\title{
Evaluación de las metodologías holmberg y konya en la mina grumintor de una malla de perforación y voladura
}

\section{Evaluation of the holmberg and konya methodologies in the drill-and-blast mesh grumintor mine}

\author{
Marco Antonio Mejía Flores. ${ }^{1}$, Gregory Guillermo Cuesta Andrade. ${ }^{2} \&$ Kevin Felipe
} Cabrera Barrera. ${ }^{3}$.

Recibido: 22-06-2021 / Revisado: 30-06-2021 /Aceptado: 19-07-2021/ Publicado: 05-08-2021

\begin{abstract}
:
DOI: https://doi.org/10.33262/concienciadigital.v4i3.1.1824

In the development of this research, the application of two drill and blast mesh methodologies Holmberg and Konya in the Grumintor mine of the Pinglio Minera Concession was analyzed, in order to estimate the replacement of the current drill and blast mesh used by the aforementioned company. It began with the field diagnosis of the mesh traditionally used, as well as the analysis of the equipment used; continuing with the study of the properties of the explosives to be used; In addition, the geological information survey of the rock was carried out to give a classification to the rocky massif, these data were processed and analyzed using computer programs managing different study variables in order to design the drilling and blasting meshes using the Holmberg methodology and the Konya methodology, and apply in the different exploration galleries. The drill and blast mesh commonly used in the mine for its exploration work are of three types and their choice is made according to the criteria, observation, and experience of the driller. The results of the application of the two methodologies will allow us to compare parameters and obtain conclusions about their applicability.

\footnotetext{
${ }^{1}$ Escuela Superior Politécnica de Chimborazo, Grupo de Investigación de Recursos Mineros e Ingeniería, marco.mejia@espoch.edu.ec; https://orcid.org/0000-0002-7566-2063

${ }^{2}$ Escuela Superior Politécnica de Chimborazo, Grupo de Investigación de Recursos Mineros e Ingeniería, gregory.cuesta@espoch.edu.ec, https://orcid.org/0000-0002-9308-0593

${ }^{3}$ Escuela Superior Politécnica de Chimborazo, kevin.cabrera @ espoch.edu.ec, https://orcid.org/0000-00020125-2010; exestudiante de la carrera geología y minas.
} 
Keywords: Optimization, Drill and blast, Holmberg, Konya.

\section{Resumen:}

En el desarrollo de esta investigación se analizó la aplicación de dos metodologías de mallas de perforación y voladura Holmberg y Konya en la mina Grumintor de la Concesión Minera Pinglio, con el fin de estimar el remplazo de la malla vigente de perforación y voladura utilizada por la compañía antes mencionada (Agrimroc, 2017). Se inició con el diagnostico de campo de la malla utilizada tradicionalmente, como también el análisis de los equipos utilizados; continuando con el estudio de las propiedades de los explosivos a utilizarse; además se realizó el levantamiento de información geológica de la roca para dar una clasificación al macizo rocoso, estos datos fueron procesados y analizados mediante programas informáticos manejando distintas variables de estudio a fin de diseñar las mallas de perforación y voladura usando la metodología de Holmberg y la metodología de Konya, y aplicar en las diferentes galerías de exploración. Las mallas de perforación y voladura usadas habitualmente en la mina para sus labores de exploración son de tres tipos y su elección se realiza según el criterio, observación, y experiencia del perforista. Los resultados de la aplicación de las dos metodologías nos permitirán comparar parámetros y obtener conclusiones sobre la aplicabilidad de estas y elegir la malla más adecuada de perforación y voladura.

Palabras Clave: Optimización, Perforación y voladura, Holmberg, Konya.

\section{Introducción}

El presente estudio tiene como finalidad evaluar el desarrollo de un diseño de una malla de perforación y voladura aplicando las metodologías de Holmberg y la de Konya en la mina Grumintor S.A. de la concesión Pinglio ubicado en la provincia del Azuay en el cantón Camilo Ponce Enríquez en el sector de San Antonio, la cual está dedicada actividad económica representada por exploración, explotación y comercialización de metales (oro, plata) la cual realiza diferentes labores para la su extracción (Cabrera, 2021). Una de las principales operaciones es la voladura, actualmente la malla de voladura se realiza de manera empírica usando un diseño basado en la experiencia de o, minas cercanas por lo tanto ha existido fallas en la voladura y principalmente no se ha realizado las operaciones basándose en un estudio técnico de campo lo cual en la actualidad debe modificarse (Agrimroc, 2017), para eso el plan de perforación y la sección transversal del impacto se completará considerando una investigación de los límites de la mina, aplicando las estrategias de Holmberg y Konya para decidir la distribución de disparo más adecuada que se aplicará en la mina Grumintor utilizando una investigación del macizo rocoso y los atributos de los explosivos.

\section{Metodología}

\section{Ubicación y posición geográfica}

El sitio donde se realizó la investigación es la mina GRUMINTOR, la cual es una operadora minera de la concesión Pinglio, quien a su vez forma parte del distrito 
aurífero San Gerardo y se encuentra dentro de la jurisdicción de la provincia del Azuay, cantón Camilo Ponce Enríquez, sector San Antonio (Figura 1).

\section{Figura 1}

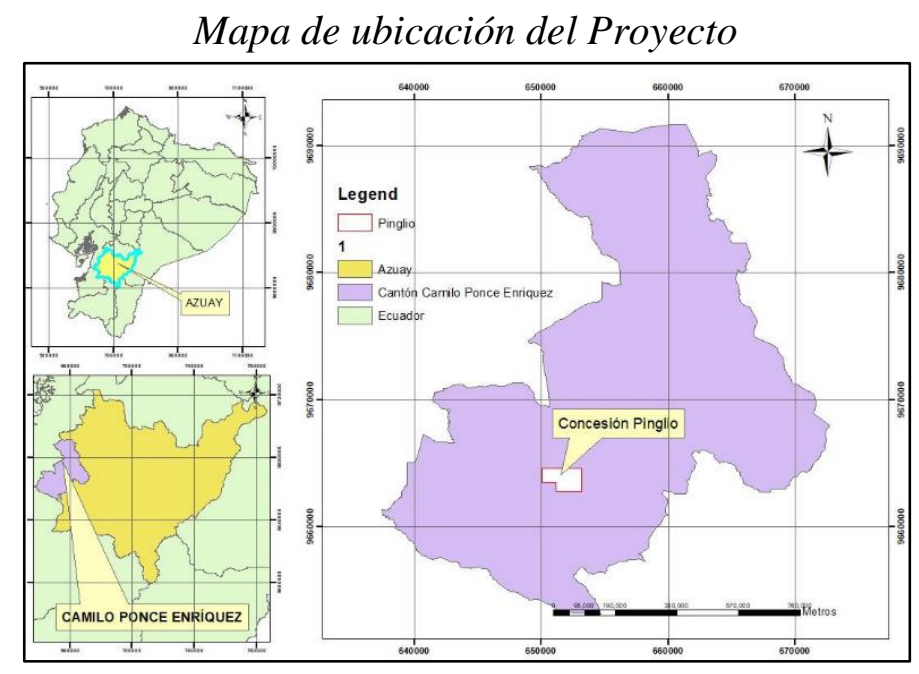

\section{Descripción Global de los frentes de explotación.}

La infraestructura básica de las labores mineras de la Mina GRUMINTOR S.A., está compuesta por una galería principal de dirección SW, además de galerías secundarias, piques, rebajes, pasos peatonales, que facilitan la extracción del material, todas estas divididas en 5 zonas de trabajo (Barton, 1974).

- la zona de la 36

- la zona del Oeste

- la zona de la Mojada

- la Zona del pique del Gallo

- La Zona del Inca

Para nuestro estudio se tomó en cuenta tres zonas: la del Inca, la G10 y de la del Gallo.

\section{Método de investigación de los frentes de explotación}

Para efectuar la evaluación de las metodologías Holmberg y Konya se empleó el método experimental, iniciando con un levantamiento minucioso de los resultados obtenidos con el uso de la malla de perforación y voladura empleada tradicionalmente. Los datos obtenidos sirven para establecer relaciones, diferencias o semejanzas, con las pruebas de perforación y voladura empleando tanto la metodología de Holmberg como de Konya y observado el comportamiento de los resultados obtenidas de cada una de las pruebas (Bach, 2016).

Primero. Se realizó el levantamiento de información geológica de la roca en el campo para formular la clasificación respectiva al macizo rocoso, en las tres zonas donde se tenía previsto aplicar los diseños de voladura mediante las metodologías planteadas de acuerdo a la investigación. 
Segundo. Se procedió a realizar una toma de muestras de roca de las galerías de la zona del Inca, de la Zona de la G10 y de la zona del Gallo para realizar ensayos de laboratorio como son el de carga puntual y el ensayo de comprensión simple, proporcionando datos que son necesarios para poder elaborar el diseño de las mallas de perforación y voladura mediante las metodologías de Holmberg y Konya, finalmente se aplicó pruebas de ensayo y error en las galerías propuestas.

Tercero. Se realizó el análisis e interpretación de resultados de la aplicación de las metodologías de Holmberg y Konya para establecer un diseño óptimo que sirva como base para futuras voladuras en la mina Grumintor, finalizando con las conclusiones y recomendaciones del caso.

\section{Las variables técnicas a tomarse en cuenta para el estudio.}

Las variables; tanto del macizo rocoso, como los explosivos y los parámetros de los equipos de perforación y voladura disponibles en la mina Grumintor, influyen para realizar el diseño de las mallas de perforación y voladura según las metodologías propuestas. Las variables consideradas fueron las siguientes (Tabla 1):

\section{Variables de Explosivo}

- Tipo

- Densidad del explosivo

- Presión de detonación

- Diámetro del explosivo

- Longitud del explosivo

- Numero de cartuchos por taladro

- Resistencia al agua

- Peso del explosivo

\section{Variables de la Roca}

- Lugar

- Tipo de la roca

- Densidad de la roca

- Resistencia a la compresión simple

- RQD

- RMR

- GSI

\section{Tabla 1}

Variables de los parámetros de los equipos de perforación

\begin{tabular}{lll}
\hline \multicolumn{1}{c}{ Parámetros } & \multicolumn{1}{c}{ Símbolo } & \multicolumn{1}{c}{ Unidades } \\
\hline Radio de baúl de túnel & $\mathrm{r}$ & $\mathrm{m}$ \\
Ancho de túnel & A Túnel & $\mathrm{m}$. \\
Alto de túnel & H Túnel & $\mathrm{m}$.
\end{tabular}




\section{Tabla 1}

Variables de los parámetros de los equipos de perforación (continuación)

\section{Parámetros}

Radio de corona o de arco de bóveda

Diámetro de taladros de producción

Diámetro de Taladro alivio

Número taladros de alivio

Ángulo de taladro de contorno

Desviación angular

Error de emboquille

Longitud de perforación por taladro

$\begin{array}{ll}\text { Símbolo } & \text { Unidades } \\ \mathrm{r} & \mathrm{m} \\ \varnothing \mathrm{o} & \mathrm{m} . \\ \varnothing 1 & \mathrm{~m} . \\ \mathrm{N} & \text { Unidades. } \\ \mathrm{Y} & \% \mathrm{~m} \\ \alpha & \mathrm{m} / \mathrm{m} . \\ \mathrm{e} & \mathrm{m} / \mathrm{m} \\ (\mathrm{L}) & \mathrm{m}\end{array}$

\section{Caracterización del macizo rocoso}

Se realizó la caracterización del macizo rocoso en la mina Grumintor mediante el sistema RMR de Bieniawski, 1989. A fin de obtener la resistencia a comprensión simple y de carga puntual se tomó una muestra de cada labor piloto sujetas al estudio de los métodos de voladura propuestos, para luego enviarlas al laboratorio; Una vez realizadas las pruebas se obtuvieron los siguientes resultados (Tabla 2):

Tabla 2

Resultados obtenidos en laboratorio de Comprensión Simple

\begin{tabular}{llccccccc}
\hline Lugar & Roca & $\begin{array}{c}\text { Área } \\
\mathbf{c m} 2\end{array}$ & $\begin{array}{c}\text { Volumen } \\
\mathbf{c m 3}\end{array}$ & $\begin{array}{c}\text { Masa } \\
\mathbf{g}\end{array}$ & $\begin{array}{c}\text { Carga } \\
\mathbf{k g}\end{array}$ & $\begin{array}{c}\text { Densidad } \\
\mathbf{g} / \mathbf{c m 3}\end{array}$ & $\begin{array}{c}\text { Esfuerzo } \\
\mathbf{k g f} / \mathbf{c m} 2\end{array}$ & $\begin{array}{c}\text { Comprensión } \\
\text { S. Mpa }\end{array}$ \\
\hline Mojada & Brecha & 31.15 & 163.52 & 455.82 & 8160 & 2.79 & 261.99 & 25.692 \\
Gallo & Dacita & 30.52 & 159.94 & 443.21 & 23720 & 2.77 & 777.1 & 76.207 \\
Inca & Andesita & 30.28 & 158.98 & 443.37 & 28980 & 2.79 & 957.04 & 93.854 \\
\hline
\end{tabular}

Los datos obtenidos en el trabajo de campo referente a los parámetros geológicos del crucero 987 se detalla en la Tabla 3:

Tabla 3

Valores tomados en el campo correspondiente a crucero 987

\begin{tabular}{lll}
\hline & Caracterización del macizo rocoso & Valoración \\
\hline Zona & El Gallo & \\
Lugar & Crucero 987 & \\
Dirección & $233 / 0$ & \\
Tipo de Roca & Dacita & 7 \\
Comprensión Simple & 76.20 & \\
Densidad & $2.77 \mathrm{~g} / \mathrm{cm} 3$ & 17 \\
R.Q.D. & 86.62 & \\
Diaclasas & & \\
\hline
\end{tabular}


Separación entre diaclasas

\section{Discontinuidades}

Longitud de la discontinuidad

Abertura de la discontinuidad

Rugosidad

Relleno

Alteraciones

\section{Agua Freática}

Caudal de agua por 10m de túnel

Orientación de discontinuidades

CLASE RMR

Clase GSI
$22 \mathrm{~cm}$

10

$1.8 \mathrm{~m}$

4

$0.001 \mathrm{~m}$

3

rugosa

5

duro

4

Ligeramente alterada

5

Ligeramente seco $\quad 10$

favorable $\quad-5$

Buena Clase II $\quad 60$

BUENA

El resumen de datos obtenidos en diferentes lecturas que se realizaron en el campo respecto a los parámetros geológicos en la Galería de Exploración 537 se detalla en la Tabla 4:

Tabla 4

Valores tomados en el campo correspondiente a la galería 537

\begin{tabular}{llr}
\hline \multicolumn{1}{c}{ Caracterización del macizo rocoso } & Valoración \\
\hline Zona & La Mojada & \\
Lugar & Galería 537 & \\
Dirección & $11 / 0$ & \\
Tipo de Roca & Brecha & \\
Densidad & $2.79 \mathrm{~g} / \mathrm{cm} 3$ & 4 \\
Comprensión Simple & $25,69 \mathrm{Mpa}$ & 17 \\
R.Q.D. & 83.32 & 10 \\
Diaclasas & & \\
Separación entre diaclasas & $34 \mathrm{~cm}$ & 4 \\
Discontinuidades & & 1 \\
Longitud de la discontinuidad & $1.1 \mathrm{~m}$ & 5 \\
Abertura de la discontinuidad & $0.003 \mathrm{~m}$ & 4 \\
Rugosidad & rugosa & 5 \\
Relleno & duro & \\
Alteraciones & Ligeramente alterada \\
Agua freática & & $\mathbf{5 2}$ \\
Caudal de agua por 10m de & Húmedo & $\mathbf{6 0}$ \\
túnel & & -5 \\
Orientación de & Medio & \\
discontinuidades & Buena Clase II & \\
CLASE RMR & BUENA & \\
CLASE GSI & &
\end{tabular}


El resumen de datos obtenidos en diferentes lecturas que se realizaron en el campo respecto a los parámetros geológicos a la galería 900, se detalla en la Tabla 5:

Tabla 5

Valores tomados en el campo correspondiente a la galería 900

\begin{tabular}{llc}
\hline \multicolumn{1}{c}{ Caracterización del macizo rocoso } & Valoración \\
\hline Zona & Galería 701 & \\
Lugar & $18 / 0$ & \\
Dirección & Andesita & \\
Tipo de Roca & $93.85 \mathrm{Mpa}$ & 7 \\
Comprensión Simple & $2.79 \mathrm{~g} / \mathrm{cm} 3$ & \\
Densidad & 87.28 & 17 \\
R.Q.D. & Diaclasas & \\
& $39 \mathrm{~cm}$ & 10 \\
Separación entre diaclasas & Discontinuidades & 6 \\
& $0.9 \mathrm{~m}$ & 1 \\
Longitud de la discontinuidad & 0.002 m & 3 \\
Abertura de la discontinuidad & Ligeramente rugosa & 4 \\
Rugosidad & duro & 5 \\
Relleno & Ligeramente alterada & \\
Alteraciones & Agua Freática & 10 \\
Caudal de agua por 10m de túnel & Nulo & -5 \\
Orientación de discontinuidades & desfavorable & $\mathbf{5 8}$ \\
CLASE RMR & Buena Clase II & $\mathbf{6 5}$ \\
CLASE GSI & BUENA & \\
\hline
\end{tabular}

Características de los explosivos Explogel III de 118 x 7

Tabla 6

\begin{tabular}{lr}
\multicolumn{2}{c}{ Características de los explosivos } \\
\hline \multicolumn{1}{c}{ Tipo } & Explogel III 1/8 x 7 \\
Densidad del explosivo & $1.05 \mathrm{~g} / \mathrm{cm}^{3}$ \\
Velocidad de Detonación & $3689 \mathrm{~m} / \mathrm{s}$ \\
Presión de detonación & $35722 \mathrm{~Pa}$ \\
Diámetro del Explosivo & $11 / 8 \%$ \\
Longitud del Explosivo & $7=0.1718 \mathrm{~m}$ \\
Resistencia al agua & $15 \mathrm{~min}$ \\
Peso Cartucho & $119 \mathrm{~g}$ \\
Número de cartuchos por caja & $212 \mathrm{unidades}$ \\
Velocidad de detonación & $3689 \mathrm{~m} / \mathrm{s}$ \\
Poder Rompedor & $17.50 \mathrm{~mm}$ \\
\hline
\end{tabular}

Los accesorios para la voladura utilizados es el fulminante $\mathrm{N}^{\circ} 8$, mecha lenta. 


\section{Diagnóstico de la malla de perforación y voladura existente}

En las Galerías de exploración se utiliza tres tipos de mallas de acuerdo con el estado de la roca ya sea dura, media y/o suave, el cual es determinado según el criterio del perforista y su geometría ynúmero de taladros está distribuido de la siguiente manera:

\section{En roca dura (Figura 2)}

\section{Figura 2}

Resultados del diagnóstico de la malla actual utilizada en roca dura

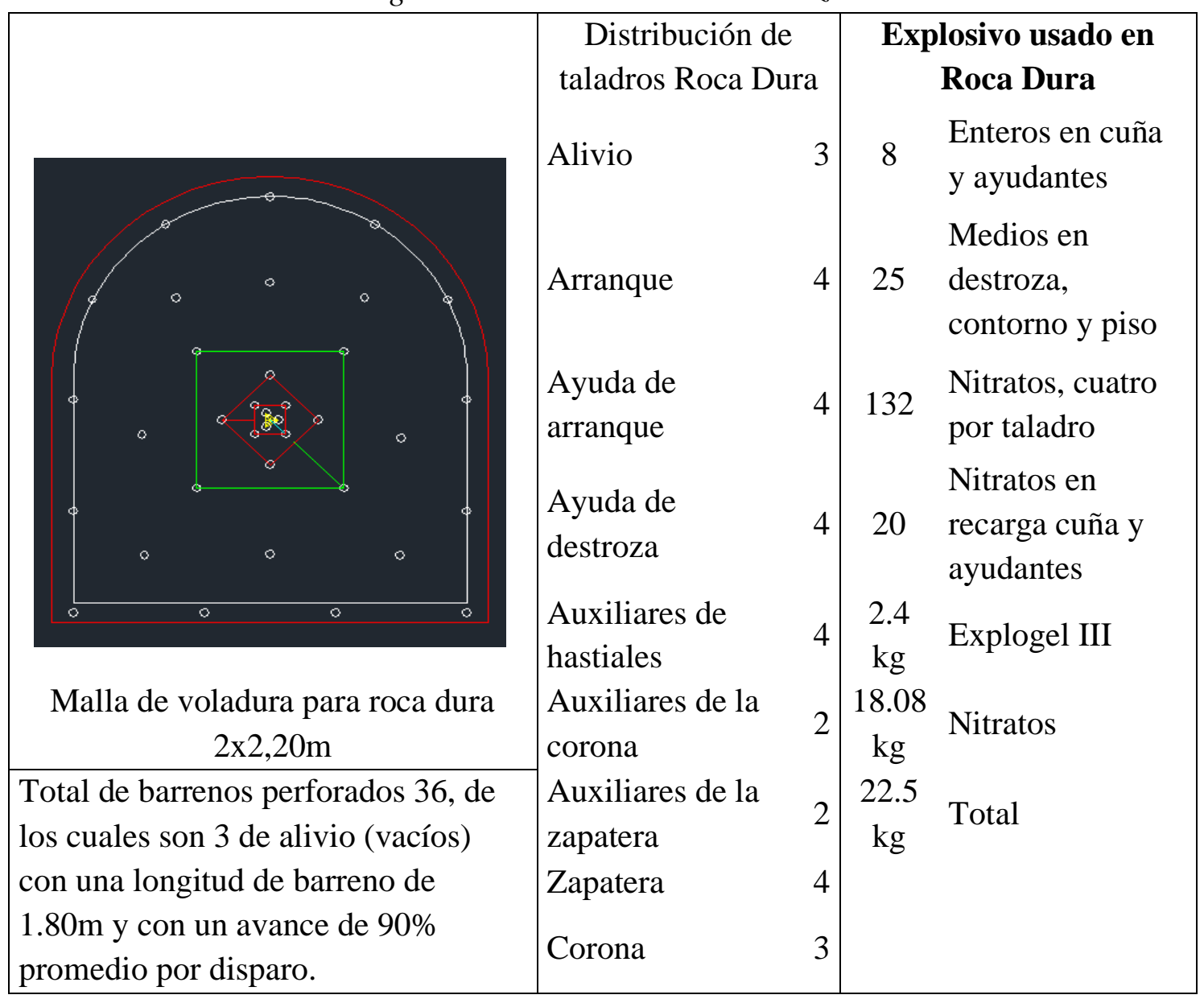




\section{En roca media (Figura 3)}

\section{Figura 3}

Resultados del diagnóstico de la malla actual utilizada en roca media

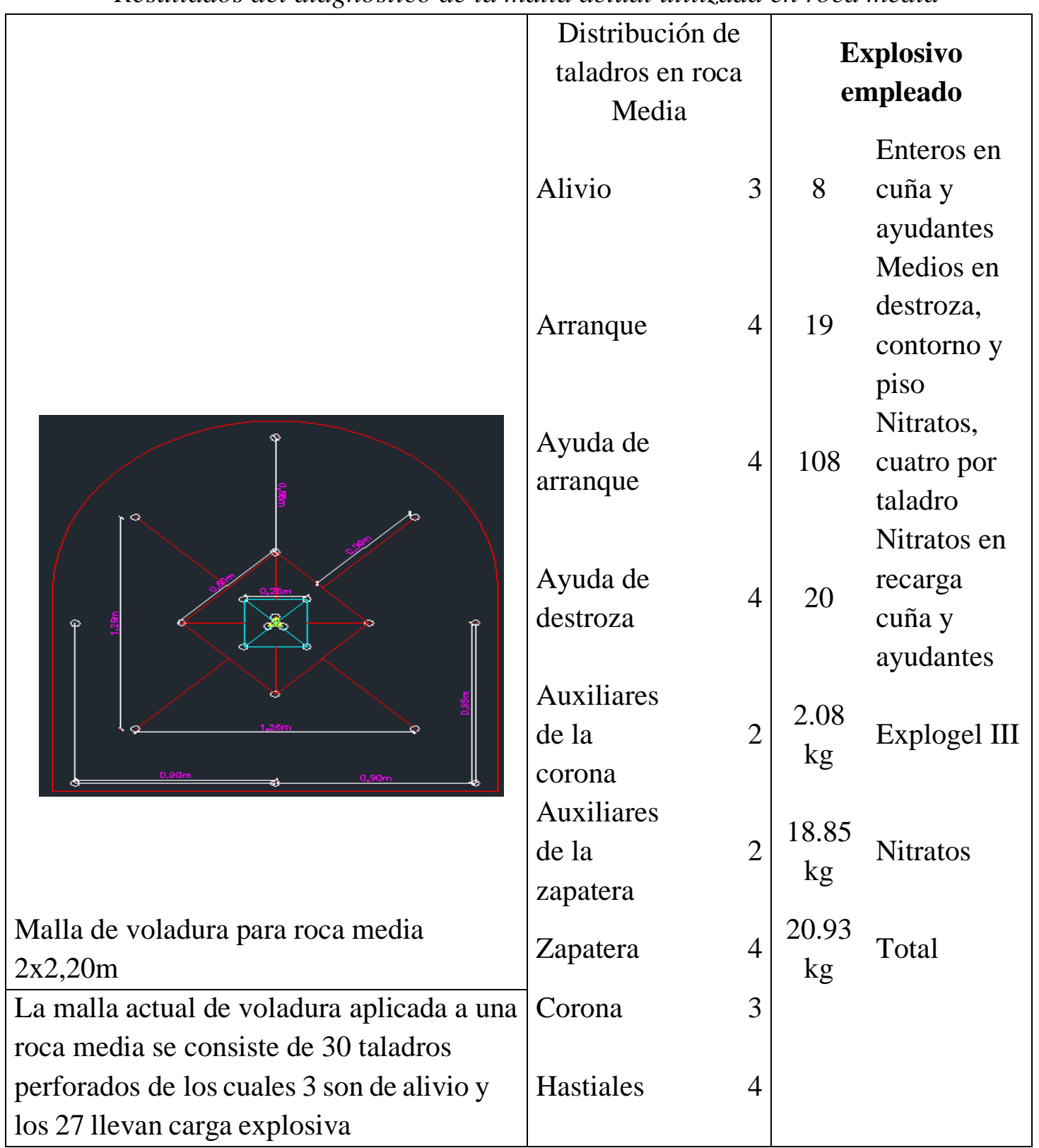




\section{Voladura en Roca suave (Figura 4)}

\section{Figura 4}

Resultados del diagnóstico de la malla actual utilizada en roca suave

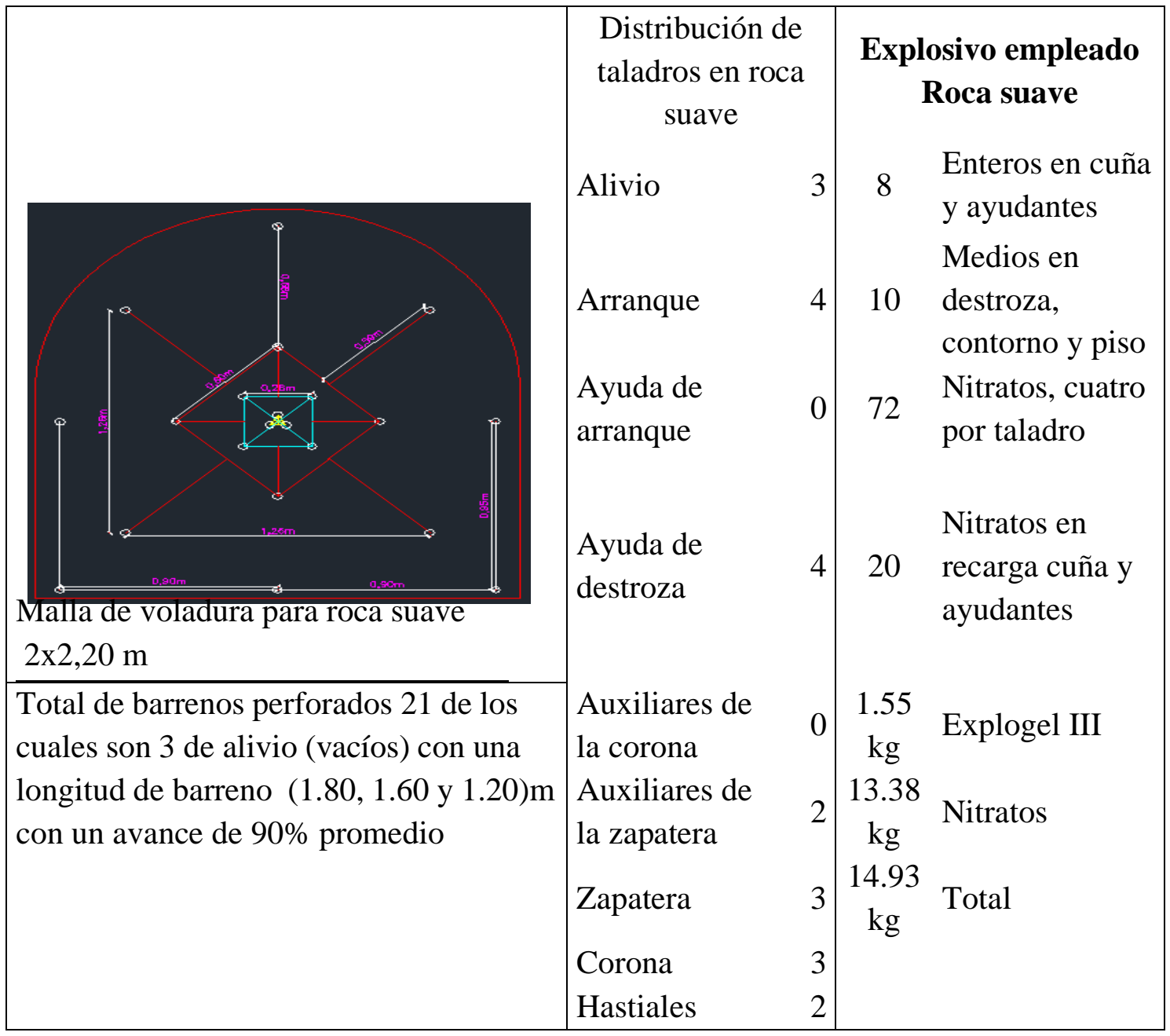

\section{Resultados}

Diseño de una malla de perforación y voladura usando la metodología de Konya.

La malla de perforación y voladura mediante la metodología de Konya considera para sus ecuaciones de cálculo los siguientes parámetros (Tabla 7):

\section{Tabla 7}

Consumo específico de explosivo para la voladura mediante Konya

Sección Taco L carga $\begin{gathered}\text { Learga } \\ \text { Explogel }\end{gathered}$ CartuchoAnfo $\begin{aligned} & \text { Cartucho } \\ & \text { Explogel }\end{aligned} \quad$ q1 $\begin{gathered}\text { Explogel } \\ \text { III (kg) }\end{gathered}$ Nitrato

\begin{tabular}{lllllllll}
\hline Primer & 0.099 & 1.611 & 0.537 & 6 & 3 & 0.73 & 1.44 & 3.55 \\
Segunda & 0.140 & 1.570 & 0.523 & 6 & 3 & 0.71 & 1.40 & 2.80 \\
\hline
\end{tabular}




\section{Tabla 7}

Consumo específico de explosivo para la voladura mediante Konya (continuación)

\begin{tabular}{lcrrrrrrr} 
Sección & Taco & L carga & $\begin{array}{r}\text { Lcarga } \\
\text { Explogel }\end{array}$ & CartuchoAnfo & $\begin{array}{r}\text { Cartucho } \\
\text { Explogel }\end{array}$ & q1 & $\begin{array}{r}\text { Explogel } \\
\text { III (kg) }\end{array}$ & Nitrato \\
\hline Tercera & 0.296 & 1.414 & 0.471 & 5 & 3 & 0.64 & 1.26 & 2.52 \\
Cuarta & 0.628 & 1.082 & 0.361 & 4 & 2 & 0.49 & 0.97 & 1.93 \\
Auxiliares & 0.707 & 1.003 & 0.167 & 5 & 1 & 0.23 & 0.45 & 2.24 \\
Piso & 0.707 & 1.003 & 0.334 & 4 & 2 & 0.45 & 0.89 & 1.79 \\
Contorno & 0.707 & 1.003 & 0.167 & 5 & 1 & 0.23 & 0.45 & 2.24 \\
Total (kg) & & & & & & & 6.86 & 17.07
\end{tabular}
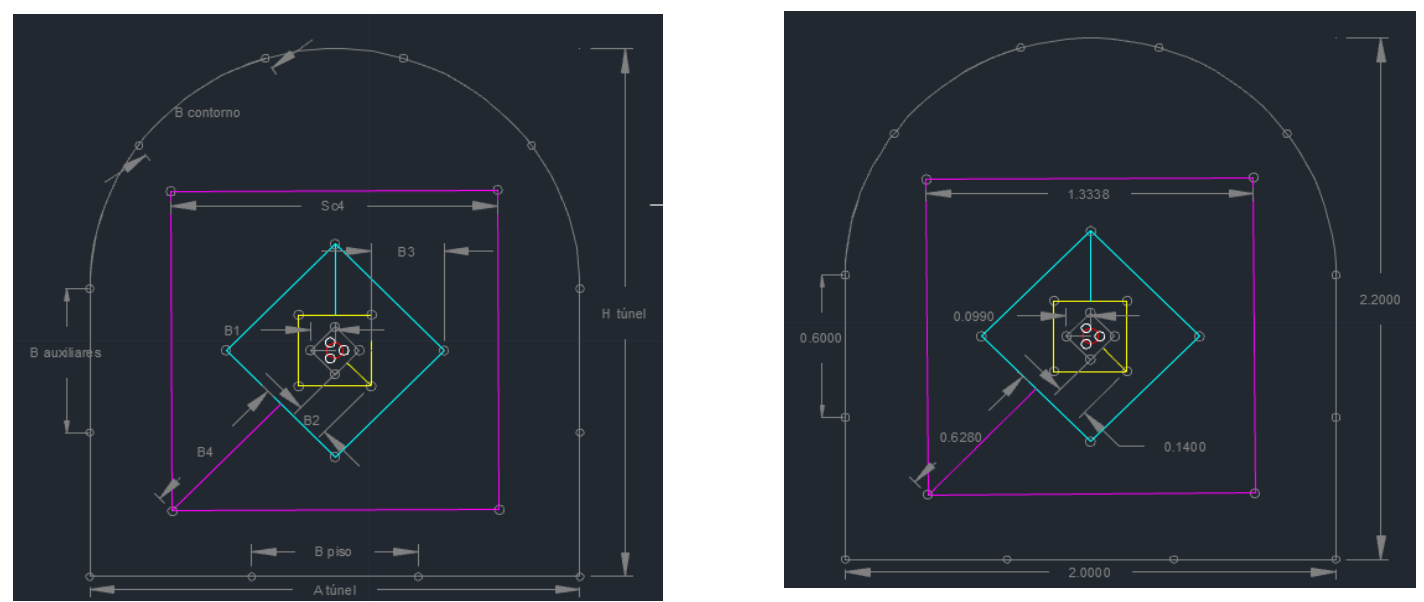

Tabla 8

Consumo específico de explosivo para la voladura mediante Konya

\begin{tabular}{|c|c|c|c|c|c|c|c|c|c|}
\hline \multicolumn{5}{|c|}{ Resultados cuñas quemadas } & \multicolumn{5}{|c|}{ Resultados según Konya } \\
\hline $\begin{array}{l}\text { Cuadro } \\
\text { No }\end{array}$ & 1 & 2 & 3 & 4 & Cuadro No & 1 & 2 & 3 & 4 \\
\hline $\mathbf{B}(\mathrm{m})$ & 0.099 & 0.140 & 0.296 & 0.628 & $\mathbf{B}(\mathrm{m})$ & 0.099 & 0.140 & 0.296 & 0.628 \\
\hline $\mathbf{R}(\mathrm{m})$ & 0.099 & 0.209 & 0.444 & 0.942 & $\mathbf{R}(\mathrm{m})$ & 0.099 & 0.209 & 0.444 & 0.942 \\
\hline Sc $(\mathrm{m})$ & 0.140 & 0.296 & 0.628 & 1.331 & Sc $(\mathrm{m})$ & 0.140 & 0.296 & 0.628 & 1.331 \\
\hline $\mathbf{T}(\mathrm{m})$ & 0.099 & 0.070 & 0.148 & 0.314 & $\mathbf{T}(\mathrm{m})$ & 0.099 & 0.070 & 0.148 & 0.314 \\
\hline $\begin{array}{l}\mathrm{Sc}>= \\
(\mathrm{L})^{\wedge}(1 / 2)\end{array}$ & 1.31 & 1.31 & 1.31 & 1.31 & $\begin{array}{l}\mathrm{Sc}>= \\
(\mathrm{L})^{\wedge}(1 / 2)\end{array}$ & 1.31 & 1.31 & 1.31 & 1.31 \\
\hline $\begin{array}{l}\mathrm{B}=\text { Distan } \\
\text { Espaciam } \\
\text { radio des } \\
\text { cuña; } \mathrm{T}=\end{array}$ & $\begin{array}{l}\text { cia barı } \\
\text { iento; } R \\
\text { le el ce } \\
\text { Taco }\end{array}$ & $\begin{array}{l}\text { enos } \\
=\text { La di } \\
\text { ntro ex }\end{array}$ & $\begin{array}{l}\text { Sc } \\
\text { stancia } \\
\text { acto de }\end{array}$ & & & & & & \\
\hline
\end{tabular}

Konya recomienda hacer el disparo de la cuña con un periodo mínimo de 50 mili segundos entre períodos. De la misma manera recomienda usar $100 \mathrm{~ms}$ de retardo para los barrenos auxiliares. Los barrenos del contorno (con voladura de recorte) se disparan con el mismo retardo. Los barrenos de piso detonan al último (Konya). 
La metodología que usa Konya para determinar los cálculos de la malla de perforación y voladura depende básicamente de las características del tipo de explosivo que se va a utilizar un sistema dearranque de cuatro secciones dependiendo de las necesidades y dimensiones de la sección de la galería por ello en las tres galerías de estudio resulta el mismo diseño ya que tienen las mismas dimensiones y se utiliza el mismo explosivo.

Diseño las mallas de perforación y voladura usando la metodología de Holmberg. Para la aplicación de la malla de perforación y voladura de las galerías se necesita los datos del explosivo, caracterización del macizo rocoso, dimensiones de la galería y las dimensiones del equipo de perforación, señalados en la siguiente Tabla 9:

Tabla 9

Resumen de parámetros para realizar el diseño en la Galería 537 (la Mojada)

\begin{tabular}{|c|c|c|c|}
\hline Labor & Símbolo & Unidades & Valor \\
\hline Zona & La Mojada & & \\
\hline GSI & - & & 60 \\
\hline RQD & - & $\%$ & 83.32 \\
\hline RMR & - & - & 52 \\
\hline Resistencia a la comprensión & - & $\mathrm{MPa}$ & 25.69 \\
\hline Densidad de la roca & $\rho r$ & $\mathrm{Tn} / \mathrm{m}^{3}$ & 2.79 \\
\hline Radio de baúl de túnel & $\mathrm{r}$ & M & 1 \\
\hline Factor de carga Suecia & $\mathrm{C}$ & $\mathrm{kg} / \mathrm{m}^{3}$ & 0.052 \\
\hline Ancho de túnel & A Túnel & $\mathrm{m}$. & 2 \\
\hline Alto de túnel & H Túnel & $\mathrm{m}$. & 2.2 \\
\hline Radio de corona o de arco de bóveda & $\mathrm{r}$ & $\mathrm{m}$ & 1 \\
\hline Diámetro de taladros de producción & $\varnothing_{0}$ & $\mathrm{~m}$. & 0.038 \\
\hline Diámetro Taladro alivio & $\varnothing 1$ & $\mathrm{~m}$. & 0.038 \\
\hline Número taladros de alivio & $\mathrm{N}$ & Unidades. & 3 \\
\hline Angulo de taladro de contorno & $\mathrm{Y}$ & $\% / \mathrm{m}$ & 3 \\
\hline Desviación angular & $\propto$ & $\mathrm{m} . / \mathrm{m}$. & 0.02 \\
\hline Error de emboquille & $\mathrm{e}$ & $\mathrm{m} . / \mathrm{m}$. & 0.02 \\
\hline $\begin{array}{l}\text { Longitud de perforación./tal (dato de } \\
\text { campo) }\end{array}$ & (L) & $\mathrm{m}$. & 1.8 \\
\hline Longitud de cartucho del explosivo & L cartucho & $\mathrm{m}$ & 0.1778 \\
\hline Valor de la constante $\mathrm{Pi}$ & $\pi$ & - & 31.416 \\
\hline Potencia relativa en peso del explosivo & PRP & (RWSanfo) & 1.05 \\
\hline Peso de un cartucho a emplear & $\begin{array}{c}\mathrm{P} \text { un } \\
\text { cartucho }\end{array}$ & $\mathrm{kg}$ & 0.119 \\
\hline Potencia relativa en peso explosivo & (RWSanfo) & $\%$ & 105 \\
\hline
\end{tabular}

Una vez aplicadas las ecuaciones con los datos obtenidos en el campo del macizo rocoso y de los instrumentos empleados se obtiene los siguientes resultados: 


\section{Resultados Galería 537 (Tabla 10, Figura 5)}

\section{Tabla 10}

Resumen de cálculos de diseño Galería 537

\begin{tabular}{|c|c|c|c|c|c|c|c|c|c|}
\hline & & & $\begin{array}{r}\text { Nún } \\
\text { tal }\end{array}$ & $\begin{array}{l}\text { nero de } \\
\text { ladros }\end{array}$ & $\begin{array}{c}\text { Densidad } \\
\text { de carga } \\
\text { lineal }\end{array}$ & & & & \\
\hline Descripción & Burden & Espaciamiento & $\begin{array}{l}\text { sin } \\
\text { carga }\end{array}$ & cargados & & cartucho/tal & kg/taladro & EplogeIII & Anfo \\
\hline $\begin{array}{l}\text { Taladro } \\
\text { arranque }\end{array}$ & 0.09 & 0.13 & 3 & 4 & 0.45 & 9 & 0.73 & 4 & 32 \\
\hline $\begin{array}{l}\text { Primer } \\
\text { cuadrante }\end{array}$ & 0.13 & 0.28 & & 4 & 0.35 & 4 & 0.47 & 4 & 12 \\
\hline $\begin{array}{l}\text { Segundo } \\
\text { cuadrante }\end{array}$ & 0.20 & 0.48 & & 4 & 0.31 & 3 & 0.41 & 4 & 8 \\
\hline $\begin{array}{l}\text { Tercer } \\
\text { cuadrante }\end{array}$ & 0.25 & 0.69 & & 4 & 0.31 & 3 & 0.41 & 4 & 8 \\
\hline $\begin{array}{l}\text { Ayuda } \\
\text { hastiales }\end{array}$ & 0.33 & 0.48 & & 2 & 0.69 & 8 & 0.92 & 2 & 14 \\
\hline Ayuda corona & 0.46 & 0.44 & & 3 & 0.64 & 7 & 0.85 & 3 & 18 \\
\hline $\begin{array}{l}\text { Ayuda de } \\
\text { arrastre }\end{array}$ & 0.27 & 0.48 & & 3 & 0.53 & 6 & 0.70 & 3 & 15 \\
\hline Hastiales & 0.28 & 0.40 & & 4 & 0.60 & 7 & 0.80 & 3 & 25 \\
\hline Corona & 0.44 & 0.57 & & 6 & 0.13 & 1 & 0.17 & 3 & 6 \\
\hline Arrastre & 0.38 & 0.59 & & 6 & 0.51 & 6 & 0.68 & 6 & 30 \\
\hline $\begin{array}{l}\text { Total taladros } \\
\text { perforados }\end{array}$ & & & & 40 & & & & & \\
\hline $\begin{array}{l}\text { Total de } \\
\text { explosivos }\end{array}$ & & & & & & & & 36 & 168 \\
\hline $\begin{array}{l}\text { Total } \\
\text { explosivos(kg) }\end{array}$ & & & & & & & & 4.284 & 24.696 \\
\hline Total (kg) & & & & & & & & & 28.98 \\
\hline
\end{tabular}

Figura 5

Malla de perforación y voladura mediante Holmberg en la Galería 5

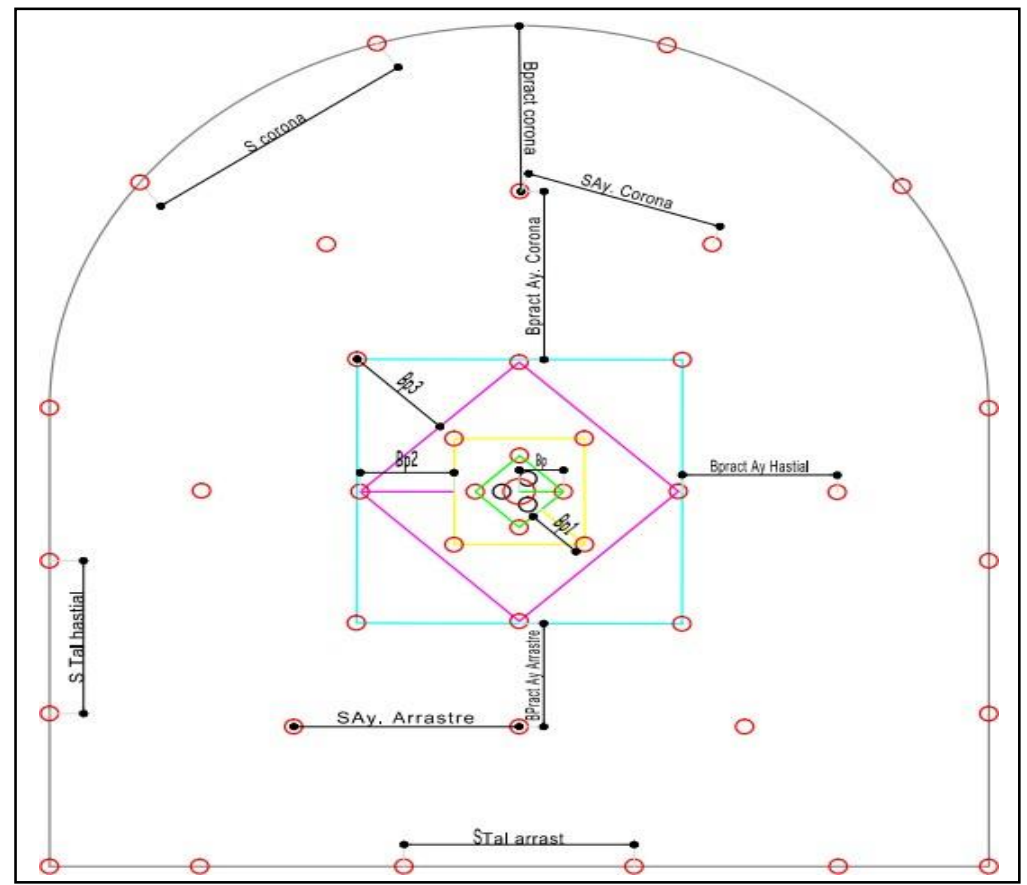




\section{Resultados del crucero 987}

Se recolectó en el campo los parámetros que requieren la metodología de Holmberg para el cálculo y elaboración de la malla detallados en la siguiente Tabla 11:

Tabla 11

Parámetros principales para elaborar el diseño en el crucero 987

\begin{tabular}{|c|c|c|c|}
\hline Labor & Símbolo & Unidades & Valor \\
\hline Zona & El Gallo & & \\
\hline GSI & - & & 65 \\
\hline RQD & - & $\%$ & 86.62 \\
\hline RMR & - & & 60 \\
\hline Resistencia a la comprensión & - & $\mathrm{MPa}$ & 76.2 \\
\hline Densidad de la roca & - & $\mathrm{Tn} / \mathrm{m}^{3}$ & 2.77 \\
\hline Radio de baúl de túnel & $\mathrm{r}$ & M & 1 \\
\hline Ancho de túnel & A Túnel & $\mathrm{m}$. & 2 \\
\hline Alto de túnel & H Túnel & m. & 2.2 \\
\hline Radio de corona o de arco de bóveda & $\mathrm{r}$ & $\mathrm{m}$ & 1 \\
\hline Diámetro de taladros de producción & $\varnothing 0$ & $\mathrm{~m}$. & 0.038 \\
\hline Diámetro Taladro alivio & $\varnothing 1$ & $\mathrm{~m}$. & 0.038 \\
\hline Número taladros de alivio & $\mathrm{N}$ & Unidades. & 3 \\
\hline Angulo de taladro de contorno & Y & $\% \mathrm{~m}$ & 3 \\
\hline Desviación angular & $\propto$ & $\mathrm{m} . / \mathrm{m}$ & 0.02 \\
\hline Error de emboquille & e & $\mathrm{m} . / \mathrm{m}$. & 0.02 \\
\hline $\begin{array}{l}\text { Longitud de perforación./tal (dato de } \\
\text { campo): }\end{array}$ & (L) & $\mathrm{m}$. & 1.8 \\
\hline Longitud de cartucho del explosivo & L cartucho & $\mathrm{m}$ & 0.1778 \\
\hline Valor de la constante Pi & $\pi$ & & 31.416 \\
\hline $\begin{array}{l}\text { Potencia relativa en peso del } \\
\text { explosivo utilizado I }\end{array}$ & PRP & (RWSanfo) & 1.05 \\
\hline Peso de un cartucho a emplear & $\mathrm{P}$ un cartucho & $\mathrm{kg}$ & 0.119 \\
\hline Potencia relativa en peso explosivo I & (RWSanfo) & $\%$ & 105 \\
\hline Capacidad de caja & - & unidades & 212 \\
\hline
\end{tabular}

La aplicación de la metodología de Holmberg para el diseño de la malla de perforación y voladura en el crucero 987 ubicado en la zona del Gallo nos dio los siguientes resultados (Tabla 12):

Tabla 12

Resumen de cálculos del diseño mediante Holmberg en el crucero 987

\begin{tabular}{|c|c|c|c|c|c|c|c|c|c|}
\hline \multirow{2}{*}{ Descripción } & \multirow{2}{*}{$\begin{array}{c}\text { Burden } \\
\text { practico } \\
\text { (Bp)m }\end{array}$} & \multirow{2}{*}{$\begin{array}{l}\text { Espaciamiento } \\
(\mathrm{Ah}) \mathrm{m}\end{array}$} & \multicolumn{2}{|c|}{$\begin{array}{l}\text { Número de } \\
\text { taladros }\end{array}$} & \multirow{2}{*}{$\begin{array}{l}\text { Densidad de } \\
\text { carga lineal }\end{array}$} & \multirow{2}{*}{$\begin{array}{l}\text { Cartucho } \\
\text { por } \\
\text { taladro }\end{array}$} & \multirow{2}{*}{$\begin{array}{c}\mathbf{K g} \\
\text { taladro }\end{array}$} & \multirow{2}{*}{$\begin{array}{c}\text { EM } \\
3000\end{array}$} & \multirow{2}{*}{ Anfo } \\
\hline & & & $\begin{array}{c}\text { Sin } \\
\text { carga }\end{array}$ & Cargado & & & & & \\
\hline $\begin{array}{c}\text { Taladro } \\
\text { arranque }\end{array}$ & 0.09 & 0.13 & 3 & 4 & 0.51 & 9 & 0.81 & 4 & 32 \\
\hline
\end{tabular}




\section{Tabla 12}

Resumen de cálculos del diseño mediante Holmberg en el crucero 987 (continuación)

\begin{tabular}{|c|c|c|c|c|c|c|c|c|c|}
\hline \multirow{2}{*}{ Descripción } & \multirow{2}{*}{$\begin{array}{c}\text { Burden } \\
\text { practico } \\
\text { (Bp)m }\end{array}$} & \multirow{2}{*}{$\begin{array}{l}\text { Espaciamiento } \\
\text { (Ah)m }\end{array}$} & \multicolumn{2}{|c|}{$\begin{array}{c}\text { Número de } \\
\text { taladros }\end{array}$} & \multirow{2}{*}{$\begin{array}{l}\text { Densidad de } \\
\text { carga lineal }\end{array}$} & \multirow{2}{*}{$\begin{array}{c}\text { Cartucho } \\
\text { por } \\
\text { taladro }\end{array}$} & \multirow{2}{*}{$\begin{array}{l}\text { Kg } \\
\text { taladro }\end{array}$} & \multirow{2}{*}{$\begin{array}{c}\text { EM } \\
3000\end{array}$} & \multirow{2}{*}{ Anfo } \\
\hline & & & $\begin{array}{c}\text { Sin } \\
\text { carga }\end{array}$ & Cargado & & & & & \\
\hline $\begin{array}{l}\text { 1er } \\
\text { cuadrante }\end{array}$ & 0.13 & 0.28 & & 4 & 0.39 & 4 & 0.51 & 4 & 12 \\
\hline $\begin{array}{l}2 \text { do } \\
\text { cuadrante }\end{array}$ & 0.20 & 0.48 & & 4 & 0.35 & 4 & 0.45 & 4 & 12 \\
\hline $\begin{array}{l}\text { 3er } \\
\text { cuadrante }\end{array}$ & 0.25 & 0.69 & & 4 & 0.34 & 4 & 0.45 & 4 & 12 \\
\hline $\begin{array}{l}\text { Taladro de } \\
\text { ayuda } \\
\text { hastiales }\end{array}$ & 0.33 & 0.48 & & 2 & 0.76 & 8 & 1.00 & 2 & 14 \\
\hline $\begin{array}{l}\text { Taladro } \\
\text { ayuda } \\
\text { corona }\end{array}$ & 0.46 & 0.44 & & 3 & 0.73 & 8 & 0.96 & 3 & 21 \\
\hline $\begin{array}{l}\text { Taladro } \\
\text { ayuda de } \\
\text { arrastre }\end{array}$ & 0.27 & 0.48 & & 3 & 0.58 & 6 & 0.76 & 3 & 15 \\
\hline $\begin{array}{l}\text { Taladro de } \\
\text { hastiales }\end{array}$ & 0.28 & 0.40 & & 4 & 0.66 & 7 & 0.86 & 3 & 25 \\
\hline $\begin{array}{l}\text { Taladro de } \\
\text { corona }\end{array}$ & 0.44 & 0.57 & & 6 & 0.13 & 1 & 0.17 & 3 & 3 \\
\hline $\begin{array}{l}\text { Taladro de } \\
\text { arrastre }\end{array}$ & 0.39 & 0.59 & & 6 & 0.58 & 6 & 0.76 & 6 & 30 \\
\hline $\begin{array}{l}\text { Total de } \\
\text { taladros } \\
\text { perforados }\end{array}$ & & & 3 & 40 & & 57 & 6.73 & & \\
\hline $\begin{array}{l}\text { Total } \\
\text { explosivos }\end{array}$ & & & & & & & & & \\
\hline $\begin{array}{l}\text { Total de } \\
\text { explosivos }\end{array}$ & & & & & & & & 36 & 176 \\
\hline $\begin{array}{l}\text { Total } \\
\text { explosivos } \\
\text { (kg) }\end{array}$ & & & & & & & & 4.28 & 25.87 \\
\hline
\end{tabular}

Luego que se efectuó la aplicación del modelo matemáticode Holmberg para el crucero 987 de la zona de explotación del gallo en el nivel -5 se obtiene el siguiente diseño (Figura 6):

Figura 6

Resumen de cálculos del diseño mediante Holmberg en el crucero 987

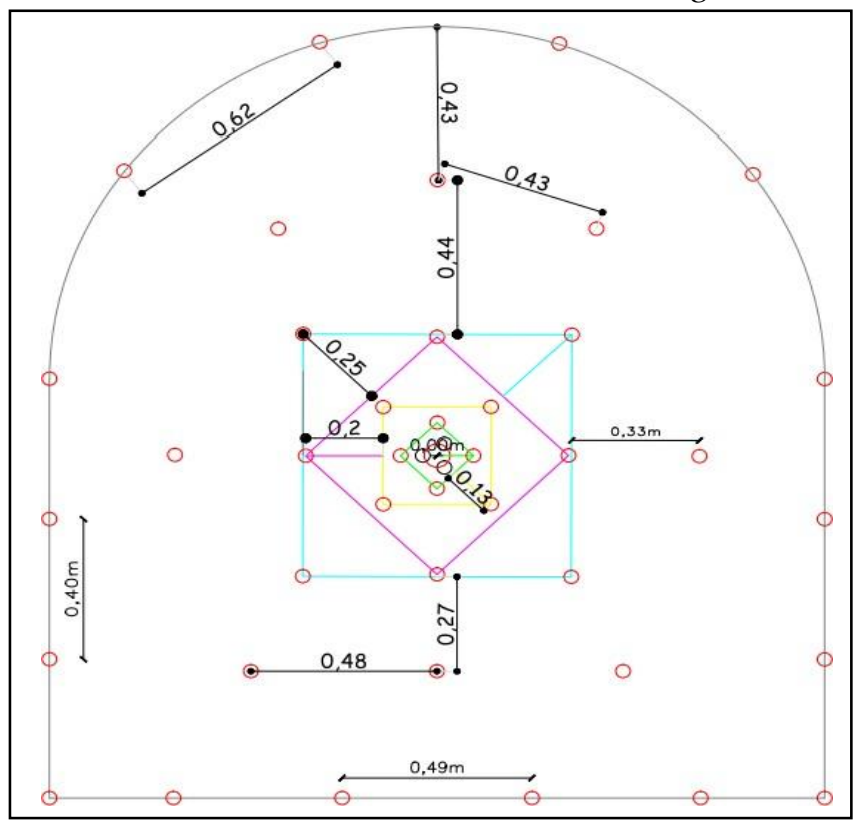




\section{Resultados la Galería de exploración 701 (Tabla 13, 14)}

Se recogió en el campo los parámetros que requieren la metodología de Holmberg para el cálculo y elaboración de la malla; Son detallados en la siguiente tabla:

Tabla 13

Parámetros principales para elaborar el diseño en la Galería 701

\begin{tabular}{|c|c|c|c|}
\hline Labor & Símbolo & Unidades & Valor \\
\hline Zona & El Inca & & \\
\hline GSI & - & - & 65 \\
\hline RQD & - & $\%$ & 87.28 \\
\hline RMR & - & - & 58 \\
\hline Resistencia a la comprensión & - & $\mathrm{MPa}$ & 93.85 \\
\hline Densidad de la roca & - & $\mathrm{Tn} / \mathrm{m}^{3}$ & 2.79 \\
\hline Radio de baúl de túnel & $\mathrm{r}$ & $\mathrm{M}$ & 1 \\
\hline Ancho de túnel & A Túnel & $\mathrm{m}$. & 2 \\
\hline Alto de túnel & H Túnel & $\mathrm{m}$. & 2.2 \\
\hline Radio de corona o de arco de bóveda & $\mathrm{r}$ & $\mathrm{m}$ & 1 \\
\hline Diámetro de taladros de producción & $\varnothing_{0}$ & $\mathrm{~m}$. & 0.038 \\
\hline Diámetro Taladro alivio & $\varnothing 1$ & m. & 0.038 \\
\hline Número taladros de alivio & $\mathrm{N}$ & Unidades. & 3 \\
\hline Angulo de taladro de contorno & Y & $\% / \mathrm{m}$ & 3 \\
\hline Desviación angular & $a$ & $\mathrm{~m} . / \mathrm{m}$. & 0.02 \\
\hline Error de emboquille & $\mathrm{e}$ & $\mathrm{m} . / \mathrm{m}$. & 0.02 \\
\hline $\begin{array}{l}\text { Longitud de perforación./tal (dato de } \\
\text { campo): }\end{array}$ & (L) & $\mathrm{m}$. & 1.8 \\
\hline Eficiencia de perforación. ( Ep ): & Ep & $\%$ & 95.44 \\
\hline Longitud de cartucho del explosivo & L cartucho & $\mathrm{m}$ & 0.1778 \\
\hline Valor de la constante Pi & $\pi$ & - & 31.416 \\
\hline $\begin{array}{l}\text { Potencia relativa en peso del explosivo } \\
\text { utilizado }\end{array}$ & PRP & RWSanfo & 1.05 \\
\hline Peso de un cartucho a emplear & $\mathrm{P}$ un cartucho & $\mathrm{kg}$ & 0.119 \\
\hline Potencia relativa en peso explosivo & RWSanfo & $\%$ & 105 \\
\hline Capacidad de caja & - & unidades & 212 \\
\hline
\end{tabular}

Tabla 14

Resumen de cálculos de diseño Galería 701

\begin{tabular}{|c|c|c|c|c|c|c|c|c|c|c|}
\hline \multirow{2}{*}{ Descripción } & \multirow{2}{*}{$\begin{array}{c}\text { Burden } \\
\text { practico } \\
\text { (Bp)m }\end{array}$} & \multirow{2}{*}{$\begin{array}{c}\text { Espaciamiento } \\
\text { (Ah)m }\end{array}$} & \multicolumn{2}{|c|}{$\begin{array}{c}\text { Número de } \\
\text { taladros }\end{array}$} & \multirow{2}{*}{$\begin{array}{c}\text { Densidad } \\
\text { de carga } \\
\text { lineal }\end{array}$} & \multirow{2}{*}{$\begin{array}{c}\text { Peso } \\
\text { cartucho }\end{array}$} & \multirow{2}{*}{$\begin{array}{c}\text { Cartucho } \\
\text { por } \\
\text { taladro }\end{array}$} & \multirow{2}{*}{ kg/taladro } & \multirow{2}{*}{$\begin{array}{c}\text { Explogel } \\
\text { III }\end{array}$} & \multirow{2}{*}{ Anfo } \\
\hline & & & $\begin{array}{c}\text { Sin } \\
\text { carga }\end{array}$ & Cargados & & & & & & \\
\hline $\begin{array}{l}\text { taladro } \\
\text { arranque }\end{array}$ & 0.09 & 0.13 & 3 & 4 & 0.51 & & 9 & 0.82 & 4 & 32 \\
\hline 1er cuadrante & 0.13 & 0.28 & & 4 & 0.40 & & 4 & 0.52 & 4 & 12 \\
\hline
\end{tabular}




\section{Tabla 14}

Resumen de cálculos de diseño Galería 701 (continuación)

\begin{tabular}{|c|c|c|c|c|c|c|c|c|c|c|}
\hline \multirow{2}{*}{ Descripción } & \multirow{2}{*}{$\begin{array}{r}\text { Burden } \\
\text { practico } \\
(\mathbf{B p}) \mathbf{m}\end{array}$} & \multirow{2}{*}{$\begin{array}{r}\text { Espaciamiento } \\
(\mathbf{A h}) \mathbf{m}\end{array}$} & \multicolumn{2}{|c|}{$\begin{array}{c}\text { Número de } \\
\text { taladros }\end{array}$} & \multirow{2}{*}{$\begin{array}{r}\text { Densidad } \\
\text { de carga } \\
\text { lineal }\end{array}$} & \multirow{2}{*}{$\begin{array}{r}\text { Peso } \\
\text { cartucho }\end{array}$} & \multirow{2}{*}{$\begin{array}{r}\text { Cartucho } \\
\text { por } \\
\text { taladro }\end{array}$} & \multirow{2}{*}{ kg/taladro } & \multirow{2}{*}{$\begin{array}{r}\text { Explogel } \\
\text { III }\end{array}$} & \multirow{2}{*}{ Anfo } \\
\hline & & & $\begin{array}{r}\text { Sin } \\
\text { carga }\end{array}$ & Cargados & & & & & & \\
\hline $\begin{array}{l}2 \text { do } \\
\text { cuadrante }\end{array}$ & 0.20 & 0.48 & & 4 & 0.35 & & 4 & 0.46 & 4 & 12 \\
\hline 3er cuadrante & 0.25 & 0.69 & & 4 & 0.35 & & 4 & 0.46 & 4 & 12 \\
\hline $\begin{array}{l}\text { tal ayuda } \\
\text { hastiales }\end{array}$ & 0.33 & 0.48 & & 2 & 0.77 & & 8 & 1.01 & 2 & 14 \\
\hline $\begin{array}{l}\text { taladro ayuda } \\
\text { corona }\end{array}$ & 0.46 & 0.44 & & 3 & 0.74 & & 8 & 0.97 & 3 & 21 \\
\hline $\begin{array}{l}\text { taladro ayuda } \\
\text { de arrastre }\end{array}$ & 0.27 & 0.48 & & 3 & 0.59 & & 6 & 0.77 & 3 & 15 \\
\hline tal hastiales & 0.28 & 0.40 & & 4 & 0.66 & & 7 & 0.86 & 3 & 25 \\
\hline tal corona & 0.44 & 0.57 & & 6 & 0.13 & & 1 & 0.17 & 3 & 3 \\
\hline tal arrastre & 0.39 & 0.59 & & 6 & 0.59 & & 6 & 0.77 & 6 & 30 \\
\hline $\begin{array}{l}\text { total taladros } \\
\text { perforados } \\
\text { total }\end{array}$ & & & 3 & 40 & & & & & 36 & 176 \\
\hline $\begin{array}{l}\text { explosivos } \\
\text { (kg) } \\
\text { total }\end{array}$ & & & & & & & & & 4.28 & 25.87 \\
\hline $\begin{array}{l}\text { explosivos } \\
(\mathrm{Kg})\end{array}$ & & & & & & & & & & 30,16 \\
\hline$(\mathrm{kg})$ & & & & & & & & & & 30.16 \\
\hline
\end{tabular}

Luego que se efectuó la aplicación del modelo matemáticode Holmberg para la galería 701 de la zona de explotación del Inca se obtiene el siguiente diseño (Figura 7):

\section{Figura 7}

Malla de perforación y voladura en la galería 701 aplicando Holmberg

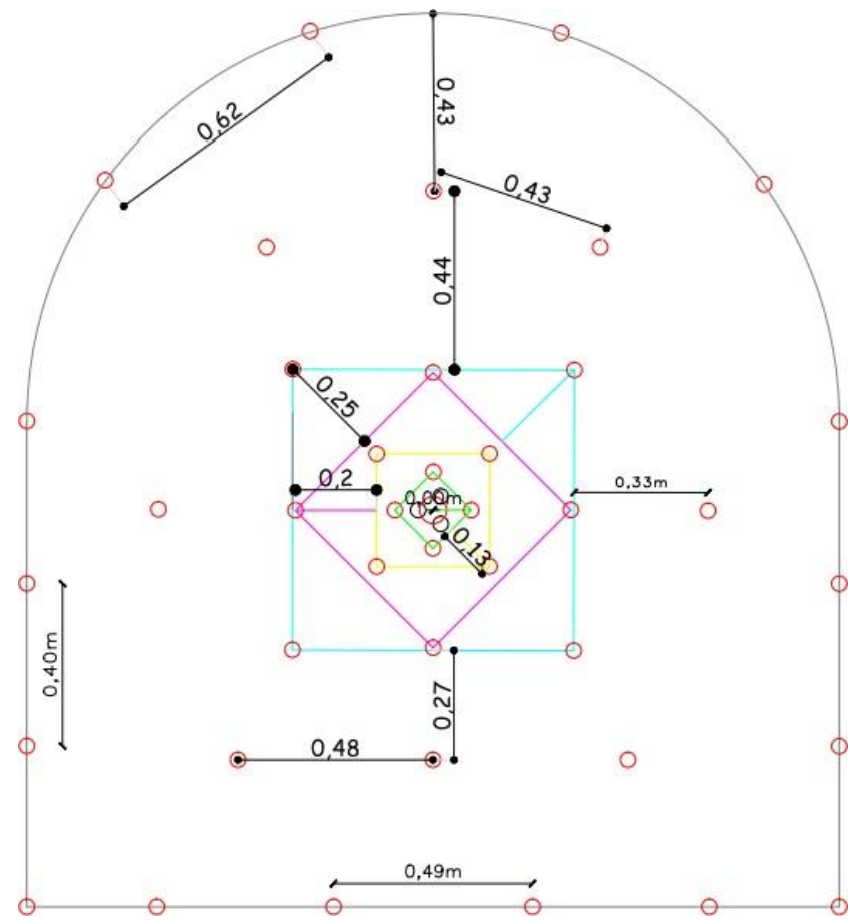




\section{Conclusiones}

- Las mallas de perforación y voladura aplicadas habitualmente en la mina Grumintor en sus labores de exploración están establecidas de acuerdo con la dureza de la roca en tres tipos, clasificándose en: roca dura, roca media y roca suave. Instaurándose una malla diferente para cada tipo de roca. Para roca dura se tiene un total de 36 barrenos perforados de los cuales son 3 de alivio, en roca media se tiene una malla de voladura que consta de 30 barrenos perforados de los cuales 27 llevan carga explosiva y 3 son de alivio, en tipo de rocas suaves la malla de voladura lleva 21 barrenos perforados de los cuales son 3 de alivio. La elección de la malla de perforación y voladura se realiza según la observación, criterio y experiencia del perforista.

- Se realizó el diseño de la malla de perforación y voladura mediante la metodología de Holmberg usando las características del macizo rocoso y se determinó que la distribución geométrica de los barrenos perforados consta de 40 barrenos que llevan carga explosiva y 3 barrenos de alivio, este diseño resultó igual en las 3 galerías de prueba, obteniendo los mismos resultados con una pequeña variación en la cantidad de explosivo utilizado.

- El resultado de la aplicación de la metodología de Holmberg es la obtención de un material volado equilibrado, no hay la necesidad de usar el martillo vibrador para reducir el tamaño de los bloques, tampoco el uso de voladuras secundarias debido a la ausencia de material no condicionado haciendo que el tiempo de limpieza mediante este método sea mucho menor. De igual manera se obtiene un perfil de la sección del túnel de forma bien definida sin necesidad de realizar voladuras secundarias en el perfil.

- El diseño y aplicación del modelo de Konya en las galerías de la mina Grumintor de la concesión Pinglio determinó una malla con solo 28 barrenos perforados de los cuales 3 son de alivio reduciendo bastante el tiempo empleado en la perforación, determinando que la distribución depende principalmente de la densidad del explosivo, el diámetro del explosivo y de la densidad de la roca tanto para barrenos auxiliares, de piso y de contorno. Para el arranque de cuatro secciones depende únicamente del diámetro de los barrenos empleados.

- Durante la aplicación de las voladuras mediante el método de Konya se presentó incidencias diarias debido a que el burden y espaciamiento son muy amplios, el material es arrancado, pero presentan gran cantidad de material no condicionado por lo tanto dificulta mucho en el ciclo de limpieza obligando al empleo de voladuras secundarias y uso de martillo vibrador para reducción de bloques muy grandes. También existe mucha variación en la definición del perfil resultante de la galería, todas estas incidencias resultan en el aumento de costos por voladura.

- En el diseño de las mallas de voladura mediante los 3 métodos aplicados se utilizaron los mismos equipos de perforación, tipo de explosivo y sus accesorios, pero al momento de hacer la elección del método óptimo tomando en cuenta los avances lineales diarios, reducción de los tiempos en el ciclo de perforación, voladura y limpieza, principalmente los costos necesarios para el uso de cada 
método y la recuperación de mineral, resultó que el más adecuado y optimo es la metodología de Holmberg para las galerías analizadas de la mina Grumintor.

- La optimización se realiza en la malla de voladura usada mediante la metodología de Holmberg, a través de las pruebas ensayo error en el campo, se determinó reducir el número de taladros en los hastiales y el uso de 4 secciones ya que según Holmberg se podría realizar más de 4 secciones.

\section{Bibliografía}

Agrimroc S.A. (2017). estudio de requerimientos técnicos para aprobación de proyecto de diseño de relaveras. Ponce Enriquez.

Albán Serrano, P. E., \& Alencastri Almeida, D. A. (2015). Plan de exportación de pulpa de pitahaya al mercado de Berlín en Alemania a través de un Comercio Justo.

Bach Fernández Tirado, J. Á. (2016). “Optimización de avance lineal, reemplazando las perforadoras jackleg por jumbo hidráulico en el proceso de perforación y voladura de la GLN1 y CRNE en Mina Consuelo de la Empresa Especializada New Horus S.A.C - Poderosa" [Universidad Nacional de Trujillo Facultad de Ingeniería Escuela Académico Profesional de Ingeniería de Minas]. Recuperado de http://www.dspace.unitru.edu.pe/bitstream/handle/UNITRU/3857/Fern\%C3\%A 1ndez\%20Tirado\%2C\%20Javier\%20\%C3\%81ngel.pdf?sequence=1\&isAllowed $=\mathrm{y}$

Barton, N Lien, R. y Lunden, J. (1974), Engineering Classification of Rock Masses for the bieniawski, Z.T. (1989). Engineering Rock Mass Clasifications. John Wiley and sons, Inc. caracterización de macizos rocosos en afloramientos. Instituto Geologico y Minero de de materiales, pp 117- 152.

Cabrera, K. (2021). Optimización de una malla de Perforación y Voladura usando las metodologías Holmberg y Konya en la mina Grumintor de la concesión Pinglio.

Deere, D. U. (1989). ROCK QUALITY DESIGNATION (RQD) AFTER TWENTY YEARS. Florida.

Desing of Tunnel Support. Rock Mechanics, Springer Verlag, vol, 6 pp 189 - 236.

Earth Science Application and Research Centre of University Hacettepe, Vol 26, pp77España. Madrid.

Explocen C.A. (s.f.). Dinamita pulverulenta sensible a fulminante No. 8 El Explogel III. Obtenido de Explocen C.A. Recuperado de https://1085-ec.all.biz/dinamitapulverulenta-sensible-a-fulminante-no-8-g436

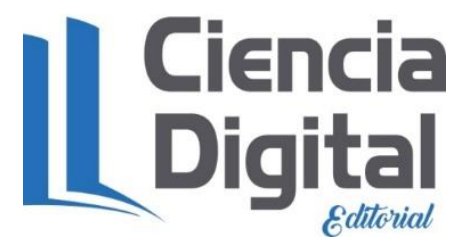




\section{PARA CITAR EL ARTÍCULO INDEXADO.}

Mejía Flores, M. A., Cuesta Andrade, G. G., \& Cabrera Barrera, K. F. (2021). Evaluación de las metodologías holmberg y konya en la mina grumintor de una malla de perforación $\begin{array}{llll}\text { y voladura. } & \text { ConcienciaDigital, } & 4(3.1), & \text { 207-226. }\end{array}$ https://doi.org/10.33262/concienciadigital.v4i3.1.1824

\section{Ciencia \\ LDigital}

El artículo que se publica es de exclusiva responsabilidad de los autores y no necesariamente reflejan el pensamiento de la Revista Conciencia Digital.

El artículo queda en propiedad de la revista y, por tanto, su publicación parcial y/o total en otro medio tiene que ser autorizado por el director de la Revista Conciencia Digital.

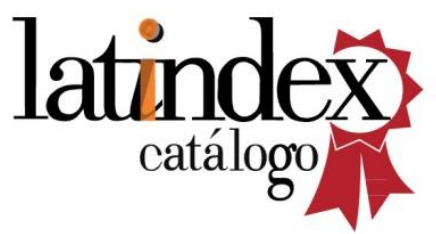

